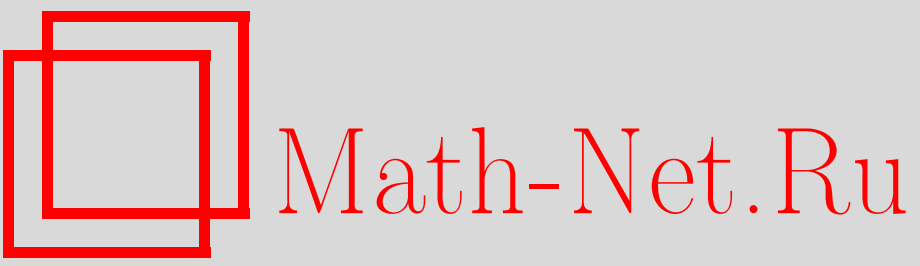

O. L. Yanushkyavichene, On bounds in limit theorems for some $U$-statistics, Teopuя вероятн. и ее примен., 2011, том 56, выпуск 4, 773-787

DOI: https://doi.org/10.4213/tvp4422

Использование Общероссийского математического портала Math-Net.Ru подразумевает, что вы прочитали и согласны с пользовательским соглашением

http://www.mathnet.ru/rus/agreement

Параметры загрузки:

IP : 54.197 .217 .227

26 апреля 2023 г., 14:40:59 


\title{
ON BOUNDS IN LIMIT THEOREMS FOR SOME $U$-STATISTICS
}

\begin{abstract}
Предлагается оценка скорости сходимости для распределения $U$-статистики второго порядка, ассоциированной с оператором Гильберта-Шмидта. Эта оценка получена для метрики Колмогорова при некоторых дополнительных условиях.

Ключевые слова и фразы: U-статистика второго порядка, предельное распределение, метрика Колмогорова, оператор Гильберта-Шмидта, собственные значения.
\end{abstract}

1. Introduction and formulation of the result. This paper pertains to the U-statistics which are currently attracting a growing attention in view of various applications (see [5]). We obtain the estimation of the convergence rate for the distribution of a second order $U$-statistic.

We begin by introducing some notation. Let $X, X_{1}, \ldots, X_{n}$ be independent identically distributed (i.i.d.) random variables taking values in a measurable space $(\Theta, \Re)$. Let $h: \Theta^{2} \rightarrow \mathbf{R}$ and $g: \Theta \rightarrow \mathbf{R}$ be real-valued measurable functions. Let $h$ be symmetric, that is, $h(x, y)=h(y, x)$ for all $x, y \in \Theta$. Consider the $U$-statistic

$$
T=T\left(X_{1}, \ldots, X_{n}\right)=n^{-1} \sum_{1 \leqslant i<k \leqslant n} h\left(X_{i}, X_{k}\right)+n^{-1 / 2} \sum_{1 \leqslant i \leqslant n} g\left(X_{i}\right),
$$

assuming that

$$
\mathbf{E} g(X)=0, \quad \mathbf{E} h(x, X)=0, \quad \text { for all } x \in \Theta .
$$

We write

$$
\gamma_{s}=\mathbf{E}|g(X)|^{s}, \quad \beta_{s}=\mathbf{E}\left|h\left(X, X_{1}\right)\right|^{s},
$$

and assume that

$$
\beta_{2}>0 \text {. }
$$

The condition $\beta_{2}>0$ ensures that the quadratic part of the statistic is not asymptotically negligible and therefore $T$ is not asymptotically normal. To

*Institute of Mathematics and Informatics, Vilnius University, Akademijos, 4, Vilnius LT-08663, Lithuania; e-mail: oljian@zebra.lt 
be more precise, the asymptotic distribution of $T$ is non-Gaussian and is given by the distribution of the random variable

$$
T_{0}=\frac{1}{2} \sum_{i \geqslant 1} q_{i}\left(\eta_{i}^{2}-1\right) .
$$

Here $\eta_{i}$ is a sequence of i.i.d. standard normal random variables and $q_{1}, q_{2}, \ldots$ denote eigenvalues of the Hilbert-Schmidt operator, say $Q$, associated with the kernel $h$ (see Section 2 for detailed definitions). Without loss of generality, we assume throughout that $\left|q_{1}\right| \geqslant\left|q_{2}\right| \geqslant \cdots$.

Denote by $\mathscr{L}(Y)$ the distribution of a random variable $Y$. Let us introduce the notation

$$
\Delta_{n}=\rho\left(\mathscr{L}\left(T\left(X_{1}, \ldots, X_{n}\right)\right), \mathscr{L}\left(T_{0}\right)\right),
$$

where $\rho$ is a Kolmogorov (or uniform) metric.

Korolyuk and Borovskikh [3] proved that $\Delta_{n}=o\left(n^{-1 / 2}\right)$ if $q_{i} \neq 0$ for all $i$. Bentkus and Götze [1] improved this estimation and found the optimal bound $\Delta_{n}=O\left(n^{-1}\right)$, under the less restrictive condition $q_{9} \neq 0$. From the method of Bentkus and Götze [1], it is clear that bounds to $\Delta_{n}$ are related to estimates of the convergence rate in the central limit theorem in Hilbert spaces. Senatov [7] constructed the lower bounds for the convergence rate on the balls in these spaces, of the order $n^{k / 12}\left|q_{1} \cdots q_{k}\right|^{1 / 2}$, for $k \leqslant 6$, if there is information that $q_{k} \neq 0$. In this article, the rate of convergence of a $U$-statistic to its limit distribution is investigated for the case, where the conditions on eigenvalues are less restrictive among all the possible ones (there is information about the first eigenvalue only). In such a case, the lower bound of Senatov is of the order $n^{-1 / 12}$.

Let

$$
\beta=\left(\beta_{18 / 5}\right)^{5 / 36}+\left(\gamma_{18 / 5}\right)^{5 / 36}<\infty .
$$

By $c$ we denote positive absolute constants that may differ from line to line and from formula to formula. The following theorem is our result.

Theorem 1. If $q_{1}>0$, then

$$
\Delta_{n} \leqslant \frac{c}{\sqrt{\left|q_{1}\right|}}\left(\frac{\beta}{n^{1 / 12}}+\frac{d}{n^{1 / 4}}\right),
$$

where $d$ is defined in (17) and depends on $q_{i}$ and $a_{i}, i=1,2, \ldots,\left(a_{i}\right.$ are some constants which we shall define in Section 2).

$\mathrm{R}$ e $\mathrm{m}$ a $\mathrm{rk}$ 1. The result of Senatov [7] allows us to suppose that the order of bound (7) is unimprovable.

In the one-dimensional case, that is, in the case where we have additional information that $q_{2}=q_{3}=\cdots=0$, the bound (7) $\Delta_{n}=O\left(n^{-1 / 12}\right)$ of our theorem improves to $\Delta_{n}=O\left(n^{-1 / 4}\right)$, and the rate $O\left(n^{-1 / 4}\right)$ is the best possible, see [9]. 
2. Special representations of bivariate $U$-statistics. Bentkus and Götze [1] suggested the following representation of bivariate $U$-statistics. Consider the measurable space $(\Theta, \mathfrak{R}, \mu)$ with a measure $\mu=\mathscr{L}(X)$. Let $L^{2}=L^{2}(\Theta, \Re, \mu)$ denote the real Hilbert space of square integrable real functions. The Hilbert-Schmidt operator is defined via

$$
Q f(x)=\int_{\Theta} h(x, y) f(y) \mu(d y), \quad f \in L^{2} .
$$

Let $\left\{e_{j}: j \geqslant 1\right\}$ denote an orthonormal complete system of eigenfunctions of $Q$ ordered by decreasing absolute values of the corresponding eigenvalues $\left|q_{1}\right| \geqslant\left|q_{2}\right| \geqslant \cdots$. Then

$$
\mathbf{E} h^{2}\left(X, X_{1}\right)=\sum_{j \geqslant 1} q_{j}^{2}<\infty, \quad h(x, y)=\sum_{j \geqslant 1} q_{j} e_{j}(x) e_{j}(y) .
$$

Consider the subspace $L^{2}(g, h) \subset L^{2}$ generated by $g$ and eigenfunctions $e_{j}$ corresponding to nonzero eigenvalues $q_{j} \neq 0$. Introducing, if necessary, a normalized eigenfunction, say $e_{0}$, such that $Q e_{0}=0$, we may assume that $e_{0}, e_{1}, \ldots$ is an orthonormal basis of $L^{2}(g, h)$. Thus, we can write

$$
g(X)=\sum_{j \geqslant 0} a_{j} e_{j}(X) \quad \text { in } L^{2}, \quad \gamma_{2}=\mathbf{E} g^{2}(X)=\sum_{j \geqslant 0} a_{j}^{2}
$$

with $a_{j}=\mathbf{E} g(X) e_{j}(X)$. It is easy to see that $\mathbf{E} e_{j}(X)=0$, for all $j$. Therefore $\left(e_{j}(X)\right)_{j \geqslant 0}$ is an orthonormal system of mean zero random variables.

Also, we shall assume throughout that all random variables and vectors are independent in aggregate, if the contrary is not clear from the context.

Let $\mathbf{R}^{\infty}$ denote the space of all real sequences $x=\left(x_{0}, x_{1}, x_{2}, \ldots\right), x_{j} \in$ $\mathbf{R}$. The Hilbert space $l_{2} \subset \mathbf{R}^{\infty}$ consists of $x \in \mathbf{R}^{\infty}$ such that

$$
|x|^{2} \stackrel{\text { def }}{=}\langle x, x\rangle, \quad|x|<\infty, \quad\langle x, y\rangle=\sum_{j \geqslant 0} x_{j} y_{j} .
$$

Consider a random vector

$$
\mathbf{X} \stackrel{\text { def }}{=}\left(e_{0}(X), e_{1}(X), \ldots\right)
$$

which takes values in $\mathbf{R}^{\infty}$. Since $\left\{e_{j}(X)\right\}_{j \geqslant 0}$ is a system of mean zero uncorrelated random variables with variances 1 , the random vector $\mathbf{X}$ has identity covariance and mean zero. Using (8) and (9) we derive

$$
h\left(X, X_{1}\right)=\left\langle Q \mathbf{X}, \mathbf{X}_{1}\right\rangle, \quad g(X)=\langle a, \mathbf{X}\rangle,
$$

where we define $Q x=\left(0, q_{1} x_{1}, q_{2} x_{2}, \ldots\right)$, for $x \in \mathbf{R}^{\infty}$, and $a=\left(a_{j}\right)_{j \geqslant 0} \in \mathbf{R}^{\infty}$. Equalities (10) allow us to assume throughout that the measurable space $\Theta$ 
is $\mathbf{R}^{\infty}$, the random variable $X$ is a random vector taking values in $\mathbf{R}^{\infty}$ with mean zero and identity covariance and that

$$
h\left(X, X_{1}\right)=\left\langle Q X, X_{1}\right\rangle, \quad g(X)=\langle a, X\rangle .
$$

In particular, without loss of generality we shall assume throughout that the kernels $h(x, y)$ and $g(x)$ are linear functions in each of their arguments.

Let $G, G_{i}, 1 \leqslant i \leqslant n$, be i.i.d. Gaussian random vectors $G_{i}=$ $\left(G_{1, i}, G_{2, i}, \ldots\right)$ with values in $\mathbf{R}^{\infty}$, where $G_{1, i}, G_{2, i}, \ldots$ denote i.i.d. standard normal random variables. We assume throughout that

$$
\mathbf{E} g(G)=0, \quad \mathbf{E} h(x, G)=0, \quad \mathbf{E} g^{2}(G)=\mathbf{E} g^{2}(X),
$$

$$
\mathbf{E} h(x, G) h(y, G)=\mathbf{E} h(x, X) h(y, X), \quad \mathbf{E} h(x, G) g(G)=\mathbf{E} h(x, X) g(X)
$$

for all $x, y$ in $\Theta$. Note that the possibility of selecting Gaussian random variables so that (11) be valid is substantiated by Bentkus and Götze in [1].

It has been also shown in [1] that it is possible to represent the statistic $T\left(G_{1}, \ldots, G_{n}\right)$ in the form

$$
T\left(G_{1}, \ldots, G_{n}\right)=n^{-1} \sum_{1 \leqslant i<k \leqslant n}\left\langle Q G_{i}, G_{k}\right\rangle+n^{-1 / 2} \sum_{1 \leqslant i \leqslant n}\left\langle a, G_{i}\right\rangle .
$$

\section{Proofs.}

3.1. Lemmas. To prove the theorem we need the following lemma, which is well known.

Lemma 1. Let $\eta$ be a standard Gaussian random variable and let $q_{1}>$ 0 . Then the distribution function $H(x)=\mathbf{P}\left\{q_{1} \eta^{2}<x\right\}$ satisfies the Lipschitz condition

$$
\sup _{x}|H(x+\varepsilon)-H(x)| \leqslant c \sqrt{\frac{\varepsilon}{q_{1}}}
$$

where $\varepsilon>0$.

The following lemma is easy to prove as well.

Lemma 2. Let $\eta$ be a standard Gaussian random variable and $q_{1}>0$. Then the following inequality is true:

$$
\sup _{x}\left|\mathbf{P}\left(q_{1} \eta_{1}^{2}-\frac{1}{n} q_{1} \eta_{1}^{2}+\frac{2}{\sqrt{n}} a_{1} \eta_{1} \leqslant x\right)-\mathbf{P}\left(q_{1} \eta_{1}^{2} \leqslant x\right)\right| \leqslant \frac{c}{\sqrt{q_{1} n}}\left(\frac{\left|a_{1}\right|}{\sqrt{q_{1}}}+1\right) .
$$

3.2. Proof of the theorem. We have to prove our theorem, i.e., the bound (7). According to the triangle inequality, we have

$$
\begin{aligned}
\Delta_{n} \leqslant & \rho\left(\mathscr{L}\left(T\left(X_{1}, \ldots, X_{n}\right)\right), \mathscr{L}\left(T\left(G_{1}, \ldots, G_{n}\right)\right)\right) \\
& +\rho\left(\mathscr{L}\left(T\left(G_{1}, \ldots, G_{n}\right)\right), \mathscr{L}\left(T_{0}\right)\right),
\end{aligned}
$$


where $G_{1}, \ldots, G_{n}$ were defined in Section 2 .

In view of (15), to prove (7) it suffices to establish that

$$
\rho\left(\mathscr{L}\left(T\left(X_{1}, \ldots, X_{n}\right)\right), \mathscr{L}\left(T\left(G_{1}, \ldots, G_{n}\right)\right)\right) \leqslant c\left|q_{1}\right|^{-1 / 2} \beta n^{-1 / 12}
$$

and

$$
\begin{aligned}
\rho\left(\mathscr{L}\left(T\left(G_{1}, \ldots, G_{n}\right)\right), \mathscr{L}\left(T_{0}\right)\right) & \leqslant \frac{c}{n^{1 / 4}}\left(\sum_{i \geqslant 1}\left|\frac{q_{i}}{q_{1}}\right|\right)^{1 / 2}+\frac{c}{n^{1 / 4}}\left(\sum_{i \geqslant 1}\left(\frac{a_{i}}{q_{1}}\right)^{2}\right)^{1 / 4} \\
& =\frac{c d}{n^{1 / 4}} .
\end{aligned}
$$

$\mathrm{R} \mathrm{e} \mathrm{m}$ a $\mathrm{rk} 2$. It is possible that the sum $\sum_{i \geqslant 1}\left|q_{i}\right|$ is divergent, i.e.,

$$
\sum_{i \geqslant 1}\left|q_{i}\right|=\infty
$$

In this case $\Delta_{n}=O\left(n^{-1}\right)$.

Indeed, if (18) holds, then $\left|q_{9}\right|>0$, since we assume that $\left|q_{1}\right| \geqslant\left|q_{2}\right| \geqslant$ $\cdots \geqslant\left|q_{9}\right| \geqslant \cdots \geqslant\left|q_{n}\right| \geqslant 0$. Then we make use of Theorem 1.1 from [1]. The theorem states that if $\left|q_{9}\right|>0$, then $\Delta_{n}=O\left(n^{-1}\right)$. That is, if the series mentioned is divergent, then estimate (7) can be replaced by the improved estimate from [1].

3.3. Proof of (16). The estimation of $\rho\left(T\left(X_{1}, \ldots, X_{n}\right), T\left(G_{1}, \ldots, G_{n}\right)\right)$ is analogous to the same one in [10].

Representation of the statistic $T\left(G_{1}, \ldots, G_{n}\right)$. It has been shown in [1] that it is possible to represent the statistic $T\left(G_{1}, \ldots, G_{n}\right)$ in the form

$$
T\left(G_{1}, \ldots, G_{n}\right)=n^{-1} \sum_{1 \leqslant i<k \leqslant n}\left\langle Q G_{i}, G_{k}\right\rangle+n^{-1 / 2} \sum_{1 \leqslant i \leqslant n}\left\langle a, G_{i}\right\rangle,
$$

where $G_{i}=\left(G_{1, i}, G_{2, i}, \ldots\right)$ and $G_{1, i}, G_{2, i}, \ldots$ denote independent standard normal random variables.

We can rewrite this expression in the form

$$
T\left(G_{1}, \ldots, G_{n}\right)=\sum_{i \geqslant 1}\left(n^{-1} q_{i} \sum_{1 \leqslant j<k \leqslant n} G_{i, j} G_{i, k}+n^{-1 / 2} \sum_{1 \leqslant j \leqslant n} a_{i} G_{i, j}\right) .
$$

It is easy to see that

$$
2 n^{-1} q_{i} \sum_{1 \leqslant j<k \leqslant n} G_{i, j} G_{i, k}=(n-1) q_{i} \bar{G}_{i}^{2}-q_{i} S_{i}^{2},
$$

where

$$
\bar{G}_{i}=n^{-1} \sum_{1 \leqslant j \leqslant n} G_{i, j} \quad \text { and } \quad S_{i}^{2}=n^{-1} \sum_{1 \leqslant j \leqslant n} G_{i, j}^{2}-\bar{G}_{i}^{2}
$$


It is well known (see [2]) that the random variables $\bar{G}_{i}$ and $S_{i}$ are independent. The random variables $\bar{G}_{i}$ have independent normal distributions and can be written in the form $\bar{G}_{i}=\eta_{i} / \sqrt{n}$, where $\eta_{i}$ are independent standard normal random variables, so we can write

$$
n^{-1} q_{i} \sum_{1 \leqslant j<k \leqslant n} G_{i, j} G_{i, k}=\frac{n-1}{n} q_{i} \eta_{i}^{2}-q_{i} S_{i}^{2} .
$$

Then

$$
\begin{aligned}
T\left(G_{1}, \ldots, G_{n}\right) & =\sum_{i \geqslant 1}\left(\frac{n-1}{2 n} q_{i} \eta_{i}^{2}-\frac{q_{i}}{2} S_{i}^{2}+a_{i} \eta_{i}\right) \\
& =\sum_{i \geqslant 1}\left(\frac{1}{2} q_{i}\left(\eta_{i}^{2}-1\right)-\frac{1}{2 n} q_{i} \eta_{i}^{2}-\frac{q_{i}}{2}\left(S_{i}^{2}-1\right)+a_{i} \eta_{i}\right) .
\end{aligned}
$$

Using of Lemma 1. Using the independence of $\eta_{i}, \eta_{j}, S_{i}, S_{j}, i \neq j$, it is easy to see, that

$$
\begin{aligned}
\sup _{x} \mid \mathbf{P}( & \left.T\left(G_{1}, \ldots, G_{n}\right) \leqslant x+\varepsilon\right)-\mathbf{P}\left(T\left(G_{1}, \ldots, G_{n}\right) \leqslant x\right) \mid \\
=\sup _{x} \mid & \mathbf{P}\left(q_{1} \eta_{1}^{2}-\frac{1}{n} q_{1} \eta_{1}^{2}+2 a_{1} \eta_{1} \leqslant x+2 \varepsilon\right) \\
& -\mathbf{P}\left(q_{1} \eta_{1}^{2}-\frac{1}{n} q_{1} \eta_{1}^{2}+2 a_{1} \eta_{1} \leqslant x\right) \mid \\
=\sup _{x} \mid & \mathbf{P}\left(\frac{q_{1}(n-1)}{n}\left(\eta_{1}+\frac{a_{1} n}{q_{1}(n-1)}\right)^{2} \leqslant x+2 \varepsilon\right) \\
& -\mathbf{P}\left(\frac{q_{1}(n-1)}{n}\left(\eta_{1}+\frac{a_{1} n}{q_{1}(n-1)}\right)^{2} \leqslant x\right) \mid .
\end{aligned}
$$

Then from Lemma 1 we get

$$
\sup _{x}\left|\mathbf{P}\left(T\left(G_{1}, \ldots, G_{n}\right) \leqslant x+\varepsilon\right)-\mathbf{P}\left(T\left(G_{1}, \ldots, G_{n}\right) \leqslant x\right)\right| \leqslant \frac{c^{\prime}}{\sqrt{\left|q_{1}\right|}} \sqrt{\varepsilon} .
$$

Using of the infinite differentiable functions. By definition we have

$$
\begin{aligned}
\rho(\mathscr{L} & \left.\left(T\left(X_{1}, \ldots, X_{n}\right)\right), \mathscr{L}\left(T\left(G_{1}, \ldots, G_{n}\right)\right)\right) \\
\quad & =\sup _{x}\left|\mathbf{P}\left(T\left(X_{1}, \ldots, X_{n}\right) \leqslant x\right)-\mathbf{P}\left(T\left(G_{1}, \ldots, G_{n}\right) \leqslant x\right)\right| .
\end{aligned}
$$

To estimate the difference

$$
\left|\mathbf{P}\left(T\left(X_{1}, \ldots, X_{n}\right) \leqslant x\right)-\mathbf{P}\left(T\left(G_{1}, \ldots, G_{n}\right) \leqslant x\right)\right|
$$

we will use infinite differentiable functions $\varphi$ such that $\left|\varphi^{(k)}(u)\right| \leqslant c_{1} / \varepsilon^{k}$, $k=1,2,3$, and $0 \leqslant \varphi(u) \leqslant 1$,

$$
\varphi(u)=1, \text { for } u \leqslant x-\varepsilon, \text { and } \varphi(u)=0, \text { for } u \geqslant x,
$$


or

$$
\varphi(u)=1, \text { for } u \leqslant x, \text { and } \varphi(u)=0, \text { for } u \geqslant x+\varepsilon
$$

Denote

$$
\Delta=\max _{\varphi}\left|\mathbf{E} \varphi\left(T\left(X_{1}, \ldots, X_{n}\right)\right)-\mathbf{E} \varphi\left(T\left(G_{1}, \ldots, G_{n}\right)\right)\right|,
$$

where max is taken over all such functions $\varphi$. Let us prove that, for any $\varepsilon>0$, we have

$$
\rho\left(\mathscr{L}\left(T\left(X_{1}, \ldots, X_{n}\right)\right), \mathscr{L}\left(T\left(G_{1}, \ldots, G_{n}\right)\right)\right) \leqslant \frac{c^{\prime}}{\sqrt{\left|q_{1}\right|}} \sqrt{\varepsilon}+\Delta .
$$

Here and in the sequel $c^{\prime}, c_{1}, c_{2}, \ldots$ are some absolute constants.

Consider $\delta^{*}=\mathbf{P}\left\{T\left(X_{1}, \ldots, X_{n}\right) \leqslant x\right\}-\mathbf{P}\left\{T\left(G_{1}, \ldots, G_{n}\right) \leqslant x\right\}$. Let us prove $(22)$ in the case $\delta^{*} \geqslant 0$. Let us take a function $\varphi$ such that (21) holds. Then

$$
\begin{aligned}
\delta^{*}= & \mathbf{E} I\left\{T\left(X_{1}, \ldots, X_{n}\right) \leqslant x\right\}-\mathbf{P}\left\{T\left(G_{1}, \ldots, G_{n}\right) \leqslant x\right\} \\
\leqslant & \left|\mathbf{E} \varphi\left(T\left(X_{1}, \ldots, X_{n}\right)\right)-\mathbf{E} \varphi\left(T\left(G_{1}, \ldots, G_{n}\right)\right)\right| \\
& +\left|\mathbf{E} \varphi\left(T\left(G_{1}, \ldots, G_{n}\right)\right)-\mathbf{P}\left\{T\left(G_{1}, \ldots, G_{n}\right) \leqslant x\right\}\right| \\
\leqslant & \Delta+\mathbf{P}\left\{x \leqslant T\left(G_{1}, \ldots, G_{n}\right) \leqslant x+\varepsilon\right\} .
\end{aligned}
$$

Using (19), we get (22).

The proof is similar if $\delta^{*}<0$, taking the function $\varphi$ that satisfies (20) instead of (21).

Estimation (22) is true for all the functions $h$ and $g$ for which conditions of Section 1 are fulfilled.

Method of induction. We write

$$
\bar{\beta}=\beta_{3}+\beta_{18 / 5}+\gamma_{3}+\gamma_{18 / 5}+1 .
$$

Let us prove that

$$
\rho\left(\mathscr{L}\left(T\left(X_{1}, \ldots, X_{n}\right)\right), \mathscr{L}\left(T\left(G_{1}, \ldots, G_{n}\right)\right)\right) \leqslant c_{2}\left|q_{1}\right|^{-1 / 2} \bar{\beta}^{1 / 6} n^{-1 / 12}
$$

for some constant $c_{2}$. We shall use induction on $n$.

Proof for $n=2$. It is easy to see that bounds (23) hold for $n=2$. Indeed, let $n=2$. Since $\rho \leqslant 1$, it is enough to show that $\left|q_{1}\right|^{-1 / 2} \bar{\beta}^{1 / 6} \geqslant 1$. We have

$$
\left|q_{1}\right|^{-1 / 2} \bar{\beta}^{1 / 6} \geqslant\left|q_{1}\right|^{-1 / 2}\left(\mathbf{E}|h|^{3}\right)^{1 / 6}=\left(q_{1}^{-2}\left(\mathbf{E}|h|^{3}\right)^{2 / 3}\right)^{1 / 4} .
$$

Using (8), we obtain

$$
\left|q_{1}\right|^{-1 / 2} \bar{\beta}^{1 / 6} \geqslant\left(q_{1}^{-2} \mathbf{E}|h|^{2}\right)^{1 / 4}=\left(q_{1}^{-2}\left(q_{1}^{2}+q_{2}^{2}+\cdots\right)\right)^{1 / 4} \geqslant 1 .
$$


The statement is proved for $n=2$.

Proof for arbitrary $n$. Assume that, for all $m \leqslant n-1$, the inequality

$$
\begin{gathered}
\rho\left(\mathscr{L}\left(T\left(X_{1}, \ldots, X_{i}, G_{i+1}, \ldots, G_{m}\right)\right), \mathscr{L}\left(T\left(G_{1}, \ldots, G_{m}\right)\right)\right) \\
\leqslant c_{2}\left|q_{1}\right|^{-1 / 2} \bar{\beta}^{1 / 6} m^{-1 / 12}
\end{gathered}
$$

is true for all $1 \leqslant i \leqslant m$ and for all the functions $h$ and $g$ in which conditions of Section 1 are fulfilled. Here $\bar{\beta}$ is the moment of functions $h$ and $g$, defined in Section 1.

Let us prove that (24) holds for $m=n$. We will prove (24) for $i=n$. By the same way it is possible to prove it for all $1 \leqslant i \leqslant n-1$.

To get the bounds (24) for $n$ we will use the following estimation:

$$
\begin{aligned}
\Delta^{*}(\varphi)= & \left|\mathbf{E} \varphi\left(T\left(X_{1}, \ldots, X_{n}\right)\right)-\mathbf{E} \varphi\left(T\left(G_{1}, \ldots, G_{n}\right)\right)\right| \\
\leqslant & \left|\mathbf{E} \varphi\left(T\left(X_{1}, \ldots, X_{n}\right)\right)-\mathbf{E} \varphi\left(T\left(X_{1}, \ldots, X_{n-1}, G_{n}\right)\right)\right| \\
& +\left|\mathbf{E} \varphi\left(T\left(X_{1}, \ldots, X_{n-1}, G_{n}\right)\right)-\mathbf{E} \varphi\left(T\left(X_{1}, \ldots, X_{n-2}, G_{n-1}, G_{n}\right)\right)\right| \\
& +\cdots+\left|\mathbf{E} \varphi\left(T\left(X_{1}, G_{2}, \ldots, G_{n}\right)\right)-\mathbf{E} \varphi\left(T\left(G_{1}, \ldots, G_{n}\right)\right)\right| \\
= & \Delta_{1, n}^{*}+\cdots+\Delta_{n, n}^{*} .
\end{aligned}
$$

Let us estimate $\Delta_{1, n}^{*}$, because we can estimate $\Delta_{i, n}^{*}$ analogously. We have

$$
\begin{aligned}
T( & \left.X_{1}, \ldots, X_{n}\right) \\
= & \frac{1}{n}\left\{h\left(X_{1}, X_{2}\right)+\sqrt{n} g\left(X_{1}\right)+\cdots+h\left(X_{1}, X_{n-1}\right)+\sqrt{n} g\left(X_{n-1}\right)\right. \\
& +h\left(X_{1}, X_{n}\right)+\sqrt{n} g\left(X_{n}\right)+h\left(X_{2}, X_{3}\right)+\cdots+h\left(X_{2}, X_{n}\right) \\
& \left.+\cdots+h\left(X_{n-2}, X_{n-1}\right)+h\left(X_{n-2}, X_{n}\right)+h\left(X_{n-1}, X_{n}\right)\right\} .
\end{aligned}
$$

We denote by $w$ the sum of all members that do not have the argument $X_{n}$ :

$$
\begin{aligned}
w=\frac{1}{n}\{ & h\left(X_{1}, X_{2}\right)+\sqrt{n} g\left(X_{1}\right)+\cdots+h\left(X_{1}, X_{n-1}\right)+\sqrt{n} g\left(X_{n-1}\right) \\
& \left.+\cdots+h\left(X_{n-2}, X_{n-1}\right)\right\}
\end{aligned}
$$

and $l_{n}$ is the sum of all members with the argument $X_{n}$ :

$$
l_{n}=\frac{1}{n}\left\{h\left(X_{1}, X_{n}\right)+\sqrt{n} g\left(X_{n}\right)+\cdots+h\left(X_{n-2}, X_{n}\right)+h\left(X_{n-1}, X_{n}\right)\right\} .
$$

Replacing $X_{n}$ by $G_{n}$, we get

$$
\begin{aligned}
T & \left(X_{1}, \ldots, X_{n-1}, G_{n}\right) \\
& =w+\frac{1}{n}\left\{h\left(X_{1}, G_{n}\right)+\sqrt{n} g\left(G_{n}\right)+\cdots+h\left(X_{n-2}, G_{n}\right)+h\left(X_{n-1}, G_{n}\right)\right\} \\
& =w+l_{n}^{*},
\end{aligned}
$$


where $l_{n}^{*}$ is obtained from $l_{n}$ replacing $X_{n}$ by $G_{n}$. We expand into the Taylor series

$$
\varphi(x+y)=\varphi(x)+\varphi^{\prime}(x) y+\frac{1}{2} \varphi^{\prime \prime}(x) y^{2}+\frac{1}{2} \mathbf{E} \varphi^{\prime \prime \prime}(x+\tau y)(1-\tau)^{2} y^{3} .
$$

Here $\tau$ is a random variable uniformly distributed in $[0,1]$ and independent of all the other random variables. Let us apply the expansion to $x=w$ and $y=l_{n}$. Write

$$
\begin{aligned}
\Delta_{1, n}^{*}= & \left|\mathbf{E} \varphi\left(T\left(X_{1}, \ldots, X_{n}\right)\right)-\mathbf{E} \varphi\left(T\left(X_{1}, \ldots, X_{n-1}, G_{n}\right)\right)\right| \\
= & \mid \mathbf{E} \varphi(w)-\mathbf{E} \varphi(w)+\mathbf{E} \varphi^{\prime}(w) l_{n}-\mathbf{E} \varphi^{\prime}(w) l_{n}^{*}+\frac{1}{2} \mathbf{E} \varphi^{\prime \prime}(w) l_{n}^{2} \\
& -\frac{1}{2} \mathbf{E} \varphi^{\prime \prime}(w)\left(l_{n}^{*}\right)^{2}+\frac{1}{2} \mathbf{E} \varphi^{\prime \prime \prime}\left(w+l_{n} \tau\right) l_{n}^{3}(1-\tau)^{2} \\
& -\frac{1}{2} \mathbf{E} \varphi^{\prime \prime \prime}\left(w+l_{n}^{*} \tau\right)\left(l_{n}^{*}\right)^{3}(1-\tau)^{2} \mid .
\end{aligned}
$$

Let $\mathbf{E}_{X_{1}, \ldots, X_{n-1}, \tau}$ be the expectation with respect to random variables $X_{1}, \ldots, X_{n-1}, \tau$, and $\mathbf{E}_{X_{n}}$ the expectation with respect to random variables $X_{n}$, and so on. Write

$$
\begin{aligned}
\Delta_{1, n}^{*}= & \mid \mathbf{E}_{X_{1}, \ldots, X_{n-1}, \tau} \varphi^{\prime}(w) \mathbf{E}_{X_{n}} l_{n}-\mathbf{E}_{X_{1}, \ldots, X_{n-1}, \tau} \varphi^{\prime}(w) \mathbf{E}_{G_{n}} l_{n}^{*} \\
& +\frac{1}{2} \mathbf{E}_{X_{1}, \ldots, X_{n-1}, \tau} \varphi^{\prime \prime}(w) \mathbf{E}_{X_{n}} l_{n}^{2}-\frac{1}{2} \mathbf{E}_{X_{1}, \ldots, X_{n-1}, \tau} \varphi^{\prime \prime}(w) \mathbf{E}_{G_{n}}\left(l_{n}^{*}\right)^{2} \\
& +\frac{1}{2} \mathbf{E} \varphi^{\prime \prime \prime}\left(w+l_{n} \tau\right) l_{n}^{3}(1-\tau)^{2}-\frac{1}{2} \mathbf{E} \varphi^{\prime \prime \prime}\left(w+l_{n}^{*} \tau\right)\left(l_{n}^{*}\right)^{3}(1-\tau)^{2} \mid
\end{aligned}
$$

Using (11), we obtain

$$
\Delta_{1, n}^{*}=\left|\frac{1}{2} \mathbf{E} \varphi^{\prime \prime \prime}\left(w+l_{n} \tau\right) l_{n}^{3}(1-\tau)^{2}-\frac{1}{2} \mathbf{E} \varphi^{\prime \prime \prime}\left(w+l_{n}^{*} \tau\right)\left(l_{n}^{*}\right)^{3}(1-\tau)^{2}\right| .
$$

Denote $\left|\mathbf{E} \varphi^{\prime \prime \prime}\left(w+l_{n} \tau\right) l_{n}^{3}(1-\tau)^{2}\right|$ by $r$ and let us estimate its value. Write

$$
l_{n}=l_{n}^{\prime}+l_{n}^{\prime \prime}
$$

with

$$
\begin{aligned}
& l_{n}^{\prime}=\frac{1}{n}\left(\sqrt{n} g\left(X_{n}\right)+\sum_{1 \leqslant k \leqslant[(n-1) / 2]} h\left(X_{k}, X_{n}\right)\right), \\
& l_{n}^{\prime \prime}=\frac{1}{n} \sum_{[(n-1) / 2]<k \leqslant n-1} h\left(X_{k}, X_{n}\right),
\end{aligned}
$$


where $[(n-1) / 2]$ is the integer part of the number $(n-1) / 2$. In a reciprocal manner, we shall define $\left(l_{n}^{*}\right)^{\prime}$ and $\left(l_{n}^{*}\right)^{\prime \prime}$. Using the inequality $(a+b)^{3} \leqslant$ $8\left(a^{3}+b^{3}\right)$ for $a>0, b>0$, we find

$$
\begin{aligned}
r \leqslant & \frac{8 c_{1}}{\varepsilon^{3}}\left(\mathbf{E} \mathbf{I}\left(x \leqslant w+\tau l_{n}^{\prime}+\tau l_{n}^{\prime \prime} \leqslant x+\varepsilon\right)\left|l_{n}^{\prime}\right|^{3}\right. \\
& \left.\quad+\mathbf{E} \mathbf{I}\left(x \leqslant w+\tau l_{n}^{\prime}+\tau l_{n}^{\prime \prime} \leqslant x+\varepsilon\right)\left|l_{n}^{\prime \prime}\right|^{3}\right) \\
= & \frac{8 c_{1}}{\varepsilon^{3}}\left(r_{1}+r_{2}\right) .
\end{aligned}
$$

We estimate only $r_{1}$, since the estimation of $r_{2}$ is similar. Now we condition $X_{1}, \ldots, X_{[(n-1) / 2]}, X_{n}, \tau$. Let $\mathbf{E}_{*}=\mathbf{E}_{X_{[(n-1) / 2]+1}, \ldots, X_{n-1}}$, then

$$
\begin{aligned}
r_{1} & =\mathbf{E}\left|l_{n}^{\prime}\right|^{3} \mathbf{E}_{*} \mathbf{I}\left(x \leqslant w+\tau l_{n}^{\prime}+\tau l_{n}^{\prime \prime} \leqslant x+\varepsilon\right) \\
& \leqslant \mathbf{E}\left|l_{n}^{\prime}\right|^{3} \sup _{x} \mathbf{P}\left\{x \leqslant w+\tau l_{n}^{\prime \prime} \leqslant x+\varepsilon\right\} \quad \text { (by the independency assumption) } \\
& =\mathbf{E}\left|l_{n}^{\prime}\right|^{3} \sup _{x} \mathbf{P}\left\{x \leqslant T^{*} \leqslant x+\varepsilon\right\},
\end{aligned}
$$

where $T^{*}=T^{*}\left(X_{[(n-1) / 2]+1}, \ldots, X_{n-1}\right)$. We derive $T^{*}$ from $w+\tau l_{n}^{\prime \prime}$, then we condition $X_{1}, \ldots, X_{[(n-1) / 2]}, X_{n}, \tau$. The corresponding function $h^{*}=h$ is the same and $g^{*}$ has the following form:

$$
g^{*}\left(X_{j}\right)=g\left(X_{j}\right)+n^{-1 / 2} \sum_{1 \leqslant k \leqslant[(n-1) / 2]} h\left(X_{k}, X_{j}\right)+n^{-1 / 2} \tau h\left(X_{n}, X_{j}\right),
$$

where $j=[(n-1) / 2]+1, \ldots, n-1$.

Using the inequalities (19), (24), we get

$$
\sup _{x} \mathbf{P}\left(x \leqslant T^{*} \leqslant x+\varepsilon\right) \leqslant \frac{c^{\prime}}{\sqrt{\left|q_{1}\right|}} \sqrt{\varepsilon}+\frac{2 c_{2}}{\sqrt{\left|q_{1}\right|}}\left(\frac{2\left(\bar{\beta}^{*}\right)^{2}}{n-1}\right)^{1 / 12},
$$

where $\bar{\beta}^{*}=\beta_{3}+\beta_{18 / 5}+\gamma_{3}^{*}+\gamma_{18 / 5}^{*}+1, \gamma_{s}^{*}=\mathbf{E}_{*}\left|g^{*}(X)\right|^{s}$.

From Theorem 20 in [6], we derive that

$$
\begin{aligned}
n^{18 / 5} \mathbf{E}\left|l_{n}^{\prime}\right|^{18 / 5} & =\mathbf{E}\left|n^{9 / 5} g\left(X_{n}\right)+\sum_{1 \leqslant k \leqslant[(n-1) / 2]} h\left(X_{k}, X_{n}\right)\right|^{18 / 5} \\
& \leqslant c_{4} n^{9 / 5}\left(\gamma_{18 / 5}+\beta_{18 / 5}\right) \leqslant c_{4} \bar{\beta} n^{9 / 5} .
\end{aligned}
$$

There are the following inequalities in [1]:

$$
\mathbf{E}|g(G)|^{s} \leqslant c_{s} \mathbf{E}|g(X)|^{s}, \quad \mathbf{E}|h(x, G)|^{s} \leqslant c_{s} \mathbf{E}|h(x, X)|^{s}, \quad s \geqslant 2,
$$

where $c_{s}$ are some constants, depending on $s$. Let us take $c_{4}$ so large that inequality (29) be true for $\left(l_{n}^{*}\right)^{\prime}$ as well. By analogy we get

$$
\begin{aligned}
n^{3} \mathbf{E}\left|l_{n}^{\prime}\right|^{3} & =\mathbf{E}\left|n^{3 / 2} g\left(X_{n}\right)+\sum_{1 \leqslant k \leqslant[(n-1) / 2]} h\left(X_{k}, X_{n}\right)\right|^{3} \\
& \leqslant c_{3} n^{3 / 2}\left(\gamma_{3}+\beta_{3}\right) .
\end{aligned}
$$


It is easy to see that

$$
\mathbf{E}\left|l_{n}^{\prime}\right|^{3} \leqslant c_{3} \bar{\beta} n^{-3 / 2} .
$$

Let us estimate $\mathbf{E}\left(\left|l_{n}^{\prime}\right|^{3}\left(\beta^{*}\right)^{1 / 6}\right)$. Using the Hölder inequality we get

$$
\begin{aligned}
& \mathbf{E}\left(\left|l_{n}^{\prime}\right|^{3}\left(\beta^{*}\right)^{1 / 6}\right) \leqslant\left(\mathbf{E}\left|l_{n}^{\prime}\right|^{18 / 5}\right)^{5 / 6}\left(\mathbf{E} \beta^{*}\right)^{1 / 6} \\
& \quad=\left(\mathbf{E}\left|l_{n}^{\prime}\right|^{18 / 5}\right)^{5 / 6}\left(\mathbf{E}\left(\beta_{3}+\beta_{18 / 5}+\left|g^{*}(X)\right|^{3}+\left|g^{*}(X)\right|^{18 / 5}+1\right)\right)^{1 / 6} .
\end{aligned}
$$

The following bounds are true:

$$
\mathbf{E}\left|g^{*}(X)\right|^{18 / 5} \leqslant c_{4}\left(\gamma_{18 / 5}+\beta_{18 / 5}\right), \quad \mathbf{E}\left|g^{*}(X)\right|^{3} \leqslant c_{3}\left(\gamma_{3}+\beta_{3}\right) .
$$

Denoting $c_{5}=\max \left(c_{4}+1, c_{3}+1\right)$, we get

$$
\mathbf{E}\left(\left|l_{n}^{\prime}\right|^{3}\left(\beta^{*}\right)^{1 / 6}\right) \leqslant c_{5}^{1 / 6} \bar{\beta}^{1 / 6}\left(\mathbf{E}\left|l_{n}^{\prime}\right|^{18 / 5}\right)^{5 / 6} \leqslant c_{5} \bar{\beta} n^{-3 / 2} .
$$

Combining (28), (31), (32) and using $\bar{\beta}>1$, we obtain

$$
r_{1} \leqslant \frac{\bar{\beta}}{\sqrt{\left|q_{1}\right|} n^{3 / 2}}\left(c^{\prime} c_{3} \sqrt{\varepsilon}+3 c_{2} c_{5} \bar{\beta}^{1 / 6}(n-1)^{-1 / 12}\right) .
$$

We can construct the same bounds for the second summand in (26). Finally we have

$$
\Delta_{1, n}^{*} \leqslant 16 \frac{c_{1} c_{5} \bar{\beta}}{\sqrt{\left|q_{1}\right|} \varepsilon^{3} n^{3 / 2}}\left(c^{\prime} \sqrt{\varepsilon}+c_{2} \bar{\beta}^{1 / 6}(n-1)^{-1 / 12}\right) .
$$

Let $\varepsilon=\delta n^{-1 / 6} \bar{\beta}^{1 / 3}$, then we can write

$$
\Delta_{1, n}^{*} \leqslant 16 c_{1}\left(\frac{c^{\prime} c_{3} \sqrt{\delta}}{\delta^{3}}+\frac{6 c_{2} c_{4}}{\delta^{3}}\right)\left|q_{1}\right|^{-1 / 2} \bar{\beta}^{1 / 6} n^{-13 / 12} .
$$

In view of (25) and (22), we obtain

$$
\begin{aligned}
\rho(\mathscr{L} & \left.\left(T\left(X_{1}, \ldots, X_{n}\right)\right), \mathscr{L}\left(T\left(G_{1}, \ldots, G_{n}\right)\right)\right) \\
& \leqslant c^{\prime}\left|q_{1}\right|^{-1 / 2} \sqrt{\varepsilon}+16 c_{1} c_{5}\left(c^{\prime} \sqrt{\delta}+3 c_{2}\right) \delta^{-3}\left|q_{1}\right|^{-1 / 2} \bar{\beta}^{1 / 6} n^{-1 / 12} \\
& =\left(c^{\prime} \sqrt{\delta}+16 c_{1} c_{5}\left(c^{\prime} \sqrt{\delta}+3 c_{2}\right) \delta^{-3}\right)\left|q_{1}\right|^{-1 / 2} \bar{\beta}^{1 / 6} n^{-1 / 12} .
\end{aligned}
$$

Choosing $\delta$ and $c_{2}$ so that the relations $112 c_{1} c_{3} \leqslant \delta^{3}, c_{2} \geqslant 2 c^{\prime} \sqrt{\delta}$ hold, we get

$$
\rho\left(\mathscr{L}\left(T\left(X_{1}, \ldots, X_{n}\right)\right), \mathscr{L}\left(T\left(G_{1}, \ldots, G_{n}\right)\right)\right) \leqslant c_{2}\left|q_{1}\right|^{-1 / 2} \bar{\beta}^{1 / 6} n^{-1 / 12} .
$$

Note that

$$
\frac{\bar{\beta}^{1 / 6}}{\left|q_{1}\right|^{1 / 2}} \leqslant \frac{\left(\beta_{3}+\gamma_{3}\right)^{1 / 6}}{\left|q_{1}\right|^{1 / 2}}+\frac{\left(\beta_{18 / 5}+\gamma_{18 / 5}\right)^{1 / 6}}{\left|q_{1}\right|^{1 / 2}}+\frac{1}{\left|q_{1}\right|^{1 / 2}} .
$$


Using of the invariance of metric $\rho$ under the linear transformations. Let $\lambda>0$ be some constant. Using the fact that the metric $\rho$ is invariant under the linear transformations, we get

$$
\begin{aligned}
\rho & \left(\mathscr{L}\left(\lambda T\left(X_{1}, \ldots, X_{n}\right)\right), \mathscr{L}\left(\lambda T\left(G_{1}, \ldots, G_{n}\right)\right)\right) \\
& =\rho\left(\mathscr{L}\left(T\left(X_{1}, \ldots, X_{n}\right)\right), \mathscr{L}\left(T\left(G_{1}, \ldots, G_{n}\right)\right)\right) \\
& \leqslant \frac{c}{\sqrt{\left|q_{1}\right|} n^{1 / 12}}\left(\left(\beta_{3}+\gamma_{3}\right)^{1 / 6}+\lambda^{1 / 10}\left(\beta_{18 / 5}+\gamma_{18 / 5}\right)^{1 / 6}+\frac{1}{\lambda^{1 / 2}}\right) .
\end{aligned}
$$

This inequality is true for all $\lambda$. Let

$$
\lambda^{1 / 10}\left(\beta_{18 / 5}+\gamma_{18 / 5}\right)^{1 / 6}=\frac{1}{\lambda^{1 / 2}} .
$$

The estimation

$$
\begin{aligned}
& \rho\left(\mathscr{L}\left(T\left(X_{1}, \ldots, X_{n}\right)\right), \mathscr{L}\left(T\left(G_{1}, \ldots, G_{n}\right)\right)\right) \\
& \quad \leqslant \frac{c}{\sqrt{\left|q_{1}\right|} n^{1 / 12}}\left(\left(\beta_{3}+\gamma_{3}\right)^{1 / 6}+\left(\beta_{18 / 5}+\gamma_{18 / 5}\right)^{5 / 36}\right)
\end{aligned}
$$

is the best one. Obviously,

$$
\left(\beta_{3}+\gamma_{3}\right)^{1 / 6} \leqslant \beta_{3}^{1 / 6}+\gamma_{3}^{1 / 6}, \quad\left(\beta_{18 / 5}+\gamma_{18 / 5}\right)^{5 / 36} \leqslant\left(\beta_{18 / 5}\right)^{5 / 36}+\left(\gamma_{18 / 5}\right)^{5 / 36} .
$$

Using the Hölder inequality, we get

$$
\beta_{3} \leqslant\left(\beta_{18 / 5}\right)^{5 / 6}, \quad \gamma_{3} \leqslant\left(\gamma_{18 / 5}\right)^{5 / 6} .
$$

From this expression we derive

$$
\rho\left(T\left(X_{1}, \ldots, X_{n}\right), T\left(G_{1}, \ldots, G_{n}\right)\right) \leqslant \frac{c}{\sqrt{\left|q_{1}\right|}} \frac{\beta}{n^{1 / 12}} .
$$

3.4. Proof of (17). Now we estimate $\rho\left(\mathscr{L}\left(T\left(G_{1}, \ldots, G_{n}\right)\right), \mathscr{L}\left(T_{0}\right)\right)$. Using of the representation of $T\left(G_{1}, \ldots, G_{n}\right)$. Obviously,

$$
\begin{aligned}
\rho\left(\mathscr{L}\left(T\left(G_{1}, \ldots, G_{n}\right)\right), \mathscr{L}\left(T_{0}\right)\right) \\
=\sup _{x} \mid \mathbf{P}\left(\sum_{i \geqslant 1}\left(\frac{1}{2} q_{i}\left(\eta_{i}^{2}-1\right)-\frac{1}{2 n} q_{i} \eta_{i}^{2}-\frac{q_{i}}{2}\left(S_{i}^{2}-1\right)+\frac{1}{\sqrt{n}} a_{i} \eta_{i}\right) \leqslant x\right) \\
\quad-\mathbf{P}\left(\frac{1}{2} \sum_{i \geqslant 1} q_{i}\left(\eta_{i}^{2}-1\right) \leqslant x\right) \mid \\
\leqslant \sup _{x} \mid \mathbf{P}\left(\sum_{i \geqslant 1}\left(\frac{1}{2} q_{i}\left(\eta_{i}^{2}-1\right)-\frac{1}{2 n} q_{i} \eta_{i}^{2}-\frac{q_{i}}{2}\left(S_{i}^{2}-1\right)+\frac{1}{\sqrt{n}} a_{i} \eta_{i}\right) \leqslant x\right)
\end{aligned}
$$




$$
\begin{aligned}
- & \mathbf{P}\left(\sum_{i \geqslant 1}\left(\frac{1}{2} q_{i}\left(\eta_{i}^{2}-1\right)-\frac{1}{2 n} q_{i} \eta_{i}^{2}+\frac{1}{\sqrt{n}} a_{i} \eta_{i}\right) \leqslant x\right) \mid \\
+\sup _{x} \mid & \mathbf{P}\left(\sum_{i \geqslant 1}\left(\frac{1}{2} q_{i}\left(\eta_{i}^{2}-1\right)-\frac{1}{2 n} q_{i} \eta_{i}^{2}+\frac{1}{\sqrt{n}} a_{i} \eta_{i}\right) \leqslant x\right) \\
- & \mathbf{P}\left(\frac{1}{2} \sum_{1 \leqslant i} q_{i}\left(\eta_{i}^{2}-1\right) \leqslant x\right) \mid=v_{1}+v_{2} .
\end{aligned}
$$

Estimation of $v_{1}$. Using the independence of $S_{i}, \eta_{i}, \eta_{j}, i \neq j$, we obtain

$$
\begin{aligned}
v_{1}= & \mid \mathbf{P}\left(\frac{1}{2} q_{1}\left(\eta_{1}^{2}-1\right)-\frac{1}{2 n} q_{1} \eta_{1}^{2}+\frac{1}{\sqrt{n}} a_{1} \eta_{1}-\sum_{i \geqslant 1} \frac{q_{i}}{2}\left(S_{i}^{2}-1\right) \leqslant x\right) \\
& -\mathbf{P}\left(\frac{1}{2} q_{1}\left(\eta_{1}^{2}-1\right)-\frac{1}{2 n} q_{1} \eta_{1}^{2}+\frac{1}{\sqrt{n}} a_{1} \eta_{1} \leqslant x\right) \mid .
\end{aligned}
$$

It is possible to rewrite this expression in the following form, since the uniform distance is invariant under the monotone transformations:

$$
v_{1}=\left|\mathbf{P}\left(\frac{1}{2} q_{1} \eta_{1}^{2}-\sum_{i \geqslant 1} \frac{q_{i}}{2}\left(S_{i}^{2}-1\right) \leqslant x\right)-\mathbf{P}\left(\frac{1}{2} q_{1} \eta_{1}^{2} \leqslant x\right)\right| .
$$

Using the independence of $S_{i}, \eta_{i}, \eta_{j}, i \neq j$, and inequality (13), we get

$$
v_{1} \leqslant c\left(\left|q_{1}\right|^{-1} \sum_{i \geqslant 1}\left|q_{i}\right| \mathbf{E}\left|S_{1}^{2}-1\right|\right)^{1 / 2} .
$$

It follows that

$$
\begin{aligned}
\left(\mathbf{E}\left|S_{1}^{2}-1\right|\right)^{1 / 2} & =\left(\mathbf{E}\left|n^{-1} \sum_{1 \leqslant j \leqslant n}\left(G_{1, j}^{2}-1\right)-\bar{G}_{1}^{2}\right|\right)^{1 / 2} \\
& \leqslant c\left(\mathbf{E}\left|n^{-1} \sum_{1 \leqslant j \leqslant n}\left(G_{1, j}^{2}-1\right)\right|\right)^{1 / 2}+\left(\mathbf{E} \bar{G}_{1}^{2}\right)^{1 / 2}
\end{aligned}
$$

Let us estimate the summands. We have $\left(\mathbf{E} \bar{G}_{1}^{2}\right)^{1 / 2}=n^{-1 / 2}\left(\mathbf{E}\left|\eta_{1}^{2}\right|\right)^{1 / 2}=$ $n^{-1 / 2}$. Also, it is easy to see that

$$
\begin{aligned}
\left(\mathbf{E}\left|n^{-1} \sum_{1 \leqslant j \leqslant n}\left(G_{1, j}^{2}-1\right)\right|\right)^{1 / 2} & =n^{-1 / 4}\left(\mathbf{E}\left|\sum_{1 \leqslant j \leqslant n} \frac{G_{1, j}^{2}-1}{\sqrt{n}}\right|\right)^{1 / 2} \\
& \leqslant n^{-1 / 4}\left(\mathbf{E}\left(\sum_{1 \leqslant j \leqslant n} \frac{G_{i, j}^{2}-1}{\sqrt{n}}\right)^{2}\right)^{1 / 4} \leqslant c n^{-1 / 4} .
\end{aligned}
$$

Consequently,

$$
v_{1} \leqslant c\left(\sum_{i \geqslant 1}\left|\frac{q_{i}}{q_{1}}\right|\right)^{1 / 2} n^{-1 / 4} .
$$


Estimation of $v_{2}$. Let us estimate the second summand in (34):

$v_{2}=\sup _{x}\left|\mathbf{P}\left(\sum_{i \geqslant 1}\left(q_{i}\left(\eta_{i}^{2}-1\right)-\frac{1}{n} q_{i} \eta_{i}^{2}+\frac{2}{\sqrt{n}} a_{i} \eta_{i}\right) \leqslant x\right)-\mathbf{P}\left(q_{i}\left(\eta_{i}^{2}-1\right) \leqslant x\right)\right|$.

Denote

$\theta=-\frac{1}{n} q_{1} \eta_{1}^{2}+\frac{2}{\sqrt{n}} a_{1} \eta_{1}, \quad Z=\sum_{i \geqslant 2} q_{i}\left(\eta_{i}^{2}-1\right), \quad Y=\sum_{i \geqslant 2}\left(-\frac{1}{n} q_{i} \eta_{i}^{2}+\frac{2}{\sqrt{n}} a_{i} \eta_{i}\right)$.

Let $F_{1}(u)=\mathbf{P}\left(q_{1}\left(\eta_{1}^{2}-1\right)+\theta \leqslant u\right), F(u)=\mathbf{P}\left(q_{1}\left(\eta_{1}^{2}-1\right) \leqslant u\right)$, then using the expression for the convolution, we get

$$
v_{2}=\sup _{x}\left|\mathbf{E} F_{1}(x-Z-Y)-\mathbf{E} F(x-Z)\right| .
$$

Using the triangle inequality we find that

$$
v_{2} \leqslant \sup _{x}\left|\mathbf{E} F_{1}(x-Z-Y)-\mathbf{E} F_{1}(x-Z)\right|+\sup _{x}\left|\mathbf{E} F_{1}(x-Z)-\mathbf{E} F(x-Z)\right| .
$$

We have

$\sup _{x}\left|\mathbf{E} F_{1}(x-Z-Y)-\mathbf{E} F_{1}(x-Z)\right| \leqslant c\left|q_{1}\right|^{-1 / 2} \mathbf{E} \sqrt{Y}=c\left|q_{1}\right|^{-1 / 2} \mathbf{E}\left(Y^{2}\right)^{1 / 4}$.

It is easy to see that $\mathbf{E}\left(Y^{2}\right)^{1 / 4} \leqslant c n^{-1 / 4}\left(\sum_{i \geqslant 2} a_{i}^{2}\right)^{1 / 4}+c n^{-1 / 2}\left(\sum_{i \geqslant 2} q_{i}^{2}\right)^{1 / 4}$. To estimate the second summand in (35) we apply Lemma 2 which yields

$$
\sup _{x}\left|\mathbf{E} F_{1}(x-Z)-\mathbf{E} F(x-Z)\right| \leqslant \frac{c}{\sqrt{\left|q_{1}\right| n}}\left(\frac{\left|a_{1}\right|}{\sqrt{\left|q_{1}\right|}}+1\right) .
$$

Finally we have

$$
v_{2} \leqslant \frac{c}{\sqrt{\left|q_{1}\right| n}}\left(\frac{\left|a_{1}\right|}{\sqrt{\left|q_{1}\right|}}+1\right)+c n^{-1 / 4}\left(\sum_{i \geqslant 2}\left(\frac{a_{i}}{q_{1}}\right)^{2}\right)^{1 / 4}+c n^{-1 / 2}\left(\sum_{i \geqslant 2}\left(\frac{q_{i}}{q_{1}}\right)^{2}\right)^{1 / 4} .
$$

Consequently,

$$
\begin{aligned}
\rho\left(T\left(G_{1}, \ldots, G_{n}\right), T_{0}\right) \leqslant & \frac{c}{n^{1 / 4}}\left(\sum_{i \geqslant 1}\left|\frac{q_{i}}{q_{1}}\right|\right)^{1 / 2}+\frac{c}{n^{1 / 4}}\left(\sum_{i \geqslant 2}\left(\frac{a_{i}}{q_{1}}\right)^{2}\right)^{1 / 4} \\
& +\frac{c}{\sqrt{\left|q_{1}\right| n}}\left(\frac{\left|a_{1}\right|}{\sqrt{\left|q_{1}\right|}}+1\right) .
\end{aligned}
$$

Using the invariance of metric $\rho$ under the linear transformations. By analogy with (33), we get

$$
\rho\left(T\left(G_{1}, \ldots, G_{n}\right), T_{0}\right) \leqslant \frac{c}{n^{1 / 4}}\left(\sum_{i \geqslant 1}\left|\frac{q_{i}}{q_{1}}\right|\right)^{1 / 2}+\frac{c}{n^{1 / 4}}\left(\sum_{i \geqslant 2}\left(\frac{a_{i}}{q_{1}}\right)^{2}\right)^{1 / 4}+\frac{c\left|a_{1}\right|}{\left|q_{1}\right| \sqrt{n}} .
$$


If $c\left|a_{1}\right| /\left(\left|q_{1}\right| \sqrt{n}\right) \geqslant 1$, then

$$
\rho\left(T\left(G_{1}, \ldots, G_{n}\right), T_{0}\right) \leqslant \frac{c}{n^{1 / 4}}\left(\sum_{i \geqslant 1}\left|\frac{q_{i}}{q_{1}}\right|\right)^{1 / 2}+\frac{c}{n^{1 / 4}}\left(\sum_{i \geqslant 1}\left(\frac{a_{i}}{q_{1}}\right)^{2}\right)^{1 / 4},
$$

because $\rho \leqslant 1$. If $c\left|a_{1}\right| /\left(\left|q_{1}\right| \sqrt{n}\right)<1$, then $\sqrt{c\left|a_{1}\right| /\left(\left|q_{1}\right| \sqrt{n}\right)} \geqslant c\left|a_{1}\right| /\left(\left|q_{1}\right| \sqrt{n}\right)$ and the statement (17) is true in both cases.

The theorem is proved.

Acknowledgments. Our results answer a question of V. Bentkus who also suggested an idea of the proof. We would like to thank V. Senatov for advice related to the construction of the lower bounds.

\section{REFERENCES}

1. Bentkus V., Götze F. Optimal bounds in non-Gaussian limit theorems for $U$ statistics. - Ann. Probab., 1999, v. 27, № 1, p. 454-521.

2. Крамер Г. Математические методы статистики. М.: Мир, 1975, 648 с.

3. Королюк В. С., Боровских Ю.В. Скорость сходимости для вырожденных функционалов Мизеса. - Теория вероятн. и ее примен., 1988, т. 33, в. 1, с. 136-146.

4. Нагаев С.А., Чеботарев В.И. Об оценках скорости сходимости в центральной предельной теореме для случайных векторов со значениями в пространстве $l_{2}$. Математический анализ и смежные вопросы математики. Новосибирск: Наука, 1978, c. $153-182$.

5. Никитин Я. Ю. Асимптотическая эффективность непараметрических критериев. М.: Наука, 1995, 238 c.

6. Петров В. В. Предельные теоремы для сумм независимых случайных величин. М.: Наука, 1987, 317 с.

7. Сенатов В.В. Качественные эффекты в оценках скорости сходимости в центральной предельной теореме в многомерных пространствах. - Тр. МИАН, 1997, т. 215 , c. $3-239$.

8. Yanushkevichiene $O$. On the rate of convergence of second-degree random polynomials. - J. Math. Sci. (New York), 1998, v. 92, № 3, p. 3955-3959.

9. Yanushkevichiene $O$. Optimal rates of convergence of second degree polynomials in several metrics. - J. Math. Sci. (New York), 2006, v. 138, № 1, p. 5472-5479.

10. Yanushkevichiene $O$. Asymptotic rate of convergence in the degenerate $U$-statistics of second order. - Banach Center Publ., 2010, v. 90, p. 275-284.

Поступила в редакцию 28.X.2010 